\title{
In-flight performance of the Absolute Scalar Magnetometer vector mode on board the Swarm satellites
}

\author{
Jean-Michel Léger ${ }^{1,2}$, Thomas Jager ${ }^{1,2^{*}}$, François Bertrand ${ }^{1,2}$, Gauthier Hulot ${ }^{3}$, Laura Brocco ${ }^{3}$, Pierre Vigneron ${ }^{3}$, \\ Xavier Lalanne ${ }^{3}$, Arnaud Chulliat ${ }^{4,5}$ and Isabelle Fratter ${ }^{6}$
}

\begin{abstract}
The role of the Absolute Scalar Magnetometer (ASM) in the European Space Agency (ESA) Swarm mission is to deliver absolute measurements of the magnetic field's strength for science investigations and in-flight calibration of the Vector Field Magnetometer (VFM). However, the ASM instrument can also simultaneously deliver vector measurements with no impact on the magnetometer's scalar performance, using a so-called vector mode. This vector mode has been continuously operated since the beginning of the mission, except for short periods of time during commissioning. Since both scalar and vector measurements are perfectly synchronous and spatially coherent, a direct assessment of the ASM vector performance can then be carried out at instrument level without need to correct for the various magnetic perturbations generated by the satellites. After a brief description of the instrument's operating principles, a thorough analysis of the instrument's behavior is presented, as well as a characterization of its environment in flight, using an alternative high sampling rate (burst) scalar mode that could be run a few days during commissioning. The ASM vector calibration process is next detailed, with some emphasis on its sensitivity to operational conditions. Finally, the evolution of the instrument's performance during the first year of the mission is presented and discussed in view of the mission's performance requirements for vector measurements.
\end{abstract}

Keywords: Swarm; He4 magnetometer; Scalar absolute magnetometer; Vector magnetometer; Vector calibration

\section{Correspondence/findings}

Introduction

ASM payload, operating principle description, and standalone performances

As the magnetic scalar reference of the ESA Swarm mission (Friis-Christensen et al. 2006), the Absolute Scalar Magnetometer (ASM) developed by CEA-LETI with the support of CNES provides continuous absolute measurements of the Earth's magnetic field strength $B_{0}$ for science purposes and in-flight vector calibration of the Vector Field Magnetometer (VFM) fluxgate instruments. Given its key role for the mission and considering the required data availability, a cold redundancy of the ASM has been implemented. Two complete and independent ASM instruments are flown onboard each of the three satellites of the Swarm

\footnotetext{
* Correspondence: thomas.jager@cea.fr

'Université Grenoble Alpes, F-38000 Grenoble, France

${ }^{2}$ CEA, LETI, MINATEC Campus, F-38054 Grenoble, France

Full list of author information is available at the end of the article
}

constellation. The ASM electronics are located within the satellite main body, while the two ASM sensor heads are mounted at the tip of the 4-m satellite boom and linked to their respective electronics, thanks to dedicated electrical and optical harnesses. The ASM is a magnetic field to frequency converter based on atomic spectroscopy of ${ }^{4} \mathrm{He}$ in its metastable level $2^{3} S_{1}$ (Kernevez et al. 1992). A radiofrequency (RF) driven resonance between the Zeeman sublevels allows a direct, absolute, and offset-free measurement of the ambient magnetic field modulus $B_{0}$, by detection of the frequency of the RF excitation signal that matches the Larmor frequency $f_{\text {Larmor }}=\gamma_{4_{H e}} B_{0}$ corresponding to the sublevel energy splitting (where $\gamma_{4_{H}} \approx 28 \mathrm{GH} / \mathrm{T}$ is the gyromagnetic ratio of ${ }^{4} \mathrm{He}$ in the $2^{3} \mathrm{~S}_{1}$ state). This resonance signal is amplified by a selective optical pumping from the $2^{3} \mathrm{~S}_{1}$ Zeeman sublevels up to the excited $2^{3} \mathrm{P}_{0}$ state, thanks to linearly polarized laser light frequency tuned to the $D_{0}$ line $\left(\lambda_{D 0}=1,082.9081 \mathrm{~nm}\right)$. To ensure isotropic performances, specific polarization 
conditions with respect to the direction of the ambient magnetic field must be maintained (i.e., angle $\theta_{\mathrm{F}}$, the angle between the light polarization and the ambient magnetic field kept at $90^{\circ}$ ). This is achieved by using a nonmagnetic piezoelectric motor inside the ASM sensor head (Guttin et al. 1994). The ASM then delivers scalar measurements with a resolution around $1 \mathrm{pT} / \sqrt{\mathrm{Hz}}$ independently of the ambient magnetic field amplitude. This represents a significant improvement compared to the characteristics of the previous Overhauser Nuclear Magnetic Resonance scalar reference sensors used on the Ørsted and CHAMP space missions (Léger et al. 2011). The maximum ASM accuracy error after all level $1 \mathrm{~b}$ corrections is of $65 \mathrm{pT}$ $(1 \sigma)$ (Jager et al. 2010, Fratter et al. 2015), well below the 150 pT $(1 \sigma)$ budget allocated to the instrument.

In the ASM's nominal scalar acquisition mode, scalar measurements are delivered at a $1-\mathrm{Hz}$ sampling rate and are low-pass filtered at $0.4 \mathrm{~Hz}$ to avoid aliasing issues. However, the intrinsic ASM scalar bandwidth is much higher (several hundreds of $\mathrm{Hz}$ ) and provides unique capabilities compared to the previous generation of scalar reference sensors. It first makes it possible to run an alternative high-rate scalar acquisition (burst) mode (250-Hz sampling with global cut-off frequency set to $100 \mathrm{~Hz}$ ), which can be used in-flight to check and assess both the noise of the instrument and external RF environmental perturbations over a wide range of frequencies. It also makes it possible to run a different (vector) mode to acquire vector measurements, using a specific set-up that superimposes three orthogonal vector modulations $\overrightarrow{b_{m i}} \cos \left(2 \pi f_{m i} t\right), \quad i=x, y, z$ onto the ambient field $\overrightarrow{B_{0}}$ (Gravrand et al. 2001, Léger et al. 2009). A real-time analysis of the scalar field measurements at the modulation frequencies $f_{m i}$ can then be used to derive the orientation of the ambient magnetic field in the vector coils frame in addition to the absolute scalar value of the field. This vector mode has been used on Swarm, and the amplitudes and frequencies of these modulations have been set to around $50 \mathrm{nT}$ and respectively 7.93, 10.99, and $12.97 \mathrm{~Hz}$. To ensure proper vector measurements, however, and avoid interferences and aliasing errors in these measurements, it is mandatory to check in-flight that no specific noise is present around these $f_{m i}$ vector modulation frequencies. During commissioning, the burst mode has thus been operated to both estimate the scalar noise level of all instruments and assess the possible presence of signals in the vicinity of the vector modulation frequencies previously selected on-ground. This check confirmed that, in this vector mode, the ASM could deliver both scalar and vector data sampled at $1 \mathrm{~Hz}$ and low-pass filtered at $0.4 \mathrm{~Hz}$, with no modification of the scalar data quality and characteristics (i.e., accuracy, resolution, timing) compared to the scalar data delivered in the scalar mode. By design, these scalar and vector measurements are then perfectly synchronized and correspond to exactly the same spatial location, thus ensuring mutual consistency. This vector mode, first developed on an optional and experimental basis for the ASM instrument, has now become the nominal mode in flight.

\section{ASM vector mode calibration process and intrinsic performance level}

Contrary to the scalar data, the raw ASM vector measurements delivered by the vector mode are not absolute. A calibration process is thus required to ensure the reconstruction of the ASM vector magnetic field in an orthonormal ASM reference frame (Gravrand et al. 2001). This calibration process only requires the estimation of six parameters (the vector components evaluations being free of any offsets), provided vector scale factors are also corrected to first order for thermal expansion and relative rotor/stator orientation effects (to account for nonsymmetrical induced errors due to sensor alignment imperfections, residual laser polarization, and fiber coupling effects). The corresponding parameters are also computed by the calibration algorithm. This algorithm takes advantage of the consistency between ASM scalar and vector measurements and uses a least squares optimization algorithm to ensure that the reconstructed vector field modulus matches the independent scalar data for a large enough set of data, the minimized difference being referred to as the scalar residual of the ASM instrument. This calibration process can thus be performed as a fully autonomous ASM task, directly using raw measurements before any correction for satellite perturbations is being introduced. Given the operation principle of the ASM vector mode, its vector resolution then linearly depends on the ratio of the ambient field to the vector modulation amplitudes. It is by design degraded by a factor of $2 \times 10^{3}$ with respect to the scalar resolution at $50 \mu \mathrm{T}$, and the expected vector resolution is thus equal to $1 \mathrm{nT} / \sqrt{ } \mathrm{Hz}$ at $B_{0}=25 \mu \mathrm{T}$.

\section{ASM in-flight commissioning results relevant for the vector mode \\ ASM instrument scalar noise level verification and in-flight perturbation identification}

To allow preliminary resolution evaluations and in-flight magnetic environment characterizations, several burst mode sessions have been performed during the commissioning phase of the ASM instruments. Figure 1 shows example noise spectra derived for the nominal ASM FM1A (FM for flight model), ASM FM2A, and ASM 
FM3A instruments on respectively Swarm Alpha, Bravo, and Charlie satellites over an acquisition period of $5 \mathrm{~s}$. At low frequencies $(f<4$ to $5 \mathrm{~Hz})$, one can predominantly observe the expected contributions of both the natural magnetic field fluctuations and satellite motion. At higher frequencies, however, these signals are much lower and the spectra directly reveal the ASM's intrinsic scalar noise level, close to $1 \mathrm{pT} / \sqrt{\mathrm{Hz}}$ in the [DC-100 Hz] band (note that by design, the ASM is free of any $1 / f$ noise, as demonstrated on-ground, thanks to numerous scalar differential measurements). These spectra also show that the vector modulation frequencies selected on-ground can indeed be used, since no natural signal could be detected around them.

An in-depth spectrogram analysis of these burst mode data also puts into light a number of additional perturbations. Expected artificial perturbations can first be seen as a peak close to the $30-\mathrm{Hz}$ frequency in Figure 1 . This peak is due to some known internal ASM RF modulation aliasing. Frequency peaks at $3.05 \mathrm{~Hz}$ and harmonics can also be seen. These correspond to known residual signatures of the ASM internal laser $D_{0}$ wavelength control loop. Fortunately, all these perturbations can be filtered and have no impact on either the scalar or the vector $1-\mathrm{Hz}$ data.

Unexpected perturbations could nevertheless also be detected. The first one, illustrated in Figure 2, is generated by the activation of the ASM sensor motor used to ensure the ASM isotropy by controlling the $\theta_{\mathrm{F}}$ angle. Each activation gives rise to rapid fluctuations of the optical coupling in the sensor head, which in turn create

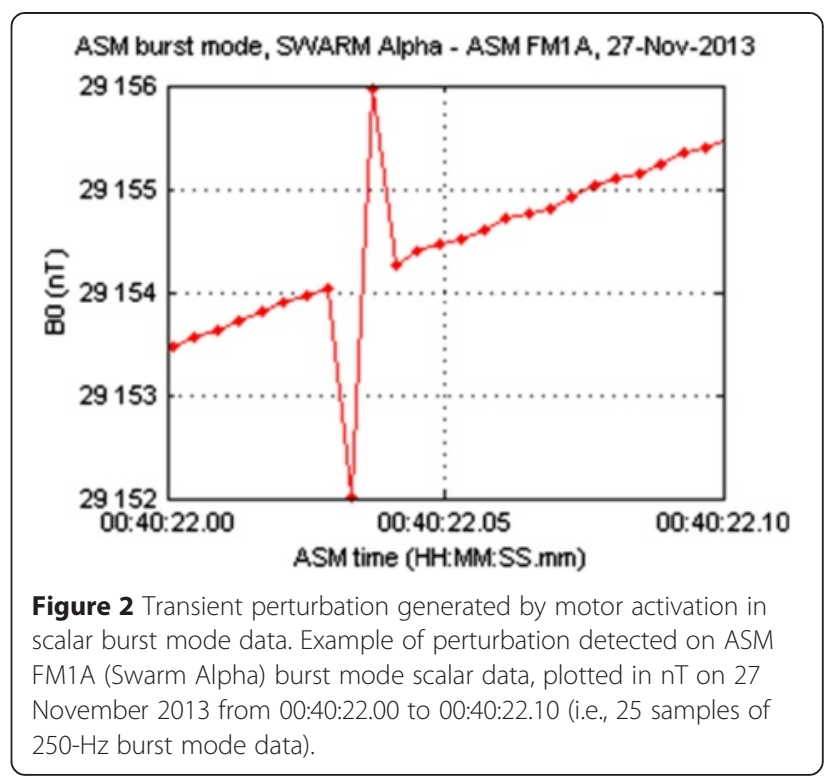

small perturbations in the ASM control loop. The resulting artifactual magnetic field transients can reach up to several nT in amplitude over a few tens of ms. Fortunately, these activations can easily be detected in the ASM housekeeping data, flagged, and corrected afterwards, using simple interpolations. Because of their very short duration, they also do not affect the $1-\mathrm{Hz}$ scalar measurements (whether acquired in scalar or vector mode), thanks to the high-order $0.4-\mathrm{Hz}$ low-pass filtering, which gets rid of them. Unfortunately, however, they do affect vector measurements performed at the vector

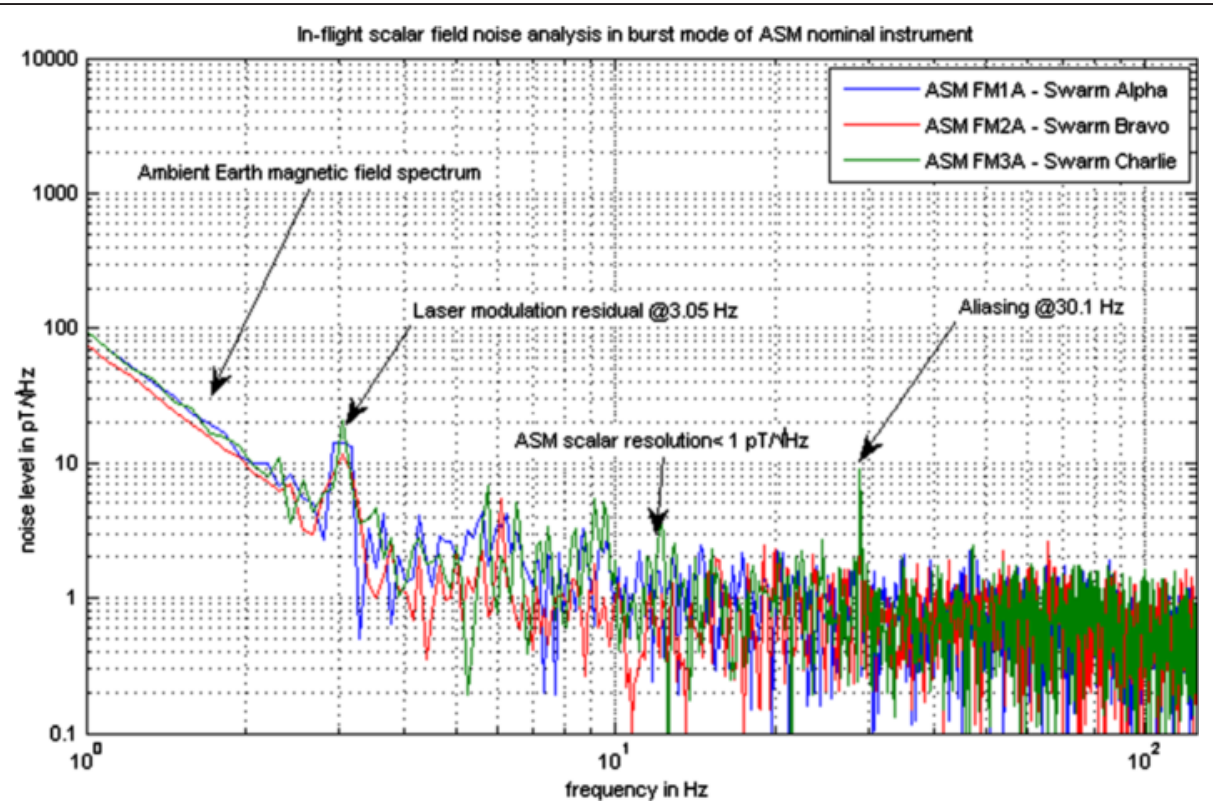

Figure 1 Burst mode scalar field noise analysis of the ASM instruments. The analysis is performed over periods of $5 \mathrm{~s}$ using 250-Hz burst mode data. The corresponding noise PSDs are plotted in $\mathrm{pT} / \sqrt{ } \mathrm{Hz}$ over the [1- to $125-\mathrm{Hz}]$ frequency band. 
modulation frequencies $f_{m i}$. This is because the vector field reconstruction process amplifies the transient perturbations by the ratio of the ambient field modulus to the vector modulation scale factors, of order $10^{3}$. Negligible $\mathrm{pT}$ range effects affecting scalar measurements are then amplified in the $\mathrm{nT}$ range in the vector measurements, despite the fact that vector measurements are also filtered by the same 0.4-Hz low-pass filter. Corresponding measurements have therefore been flagged in view of further data exploitation.

A second unexpected in-flight perturbation detected during the burst mode sessions is shown in Figure 3. After a careful analysis, this perturbation could be attributed to interferences between the $58-\mathrm{kHz}$ square signal used by the spacecraft platform to operate heaters needed for thermal control of the ASM sensors and the ASM. Each time one of the harmonics of this signal matches the ambient scalar field Larmor frequency (i.e., $f_{\text {Larmor }}=(2 k+1) \times 58 \mathrm{kHz}$ or $\left.B_{0}=(2 k+1) \times 2.071 \mu \mathrm{T}\right)$, a crosstalk occurs between these two frequencies. The geographical repartition of where these perturbations can occur is displayed in Figure 4. These fully predictable distortions are also flagged in the vector datasets.

Once properly flagged, data affected by motor activations and heater perturbations can be replaced by

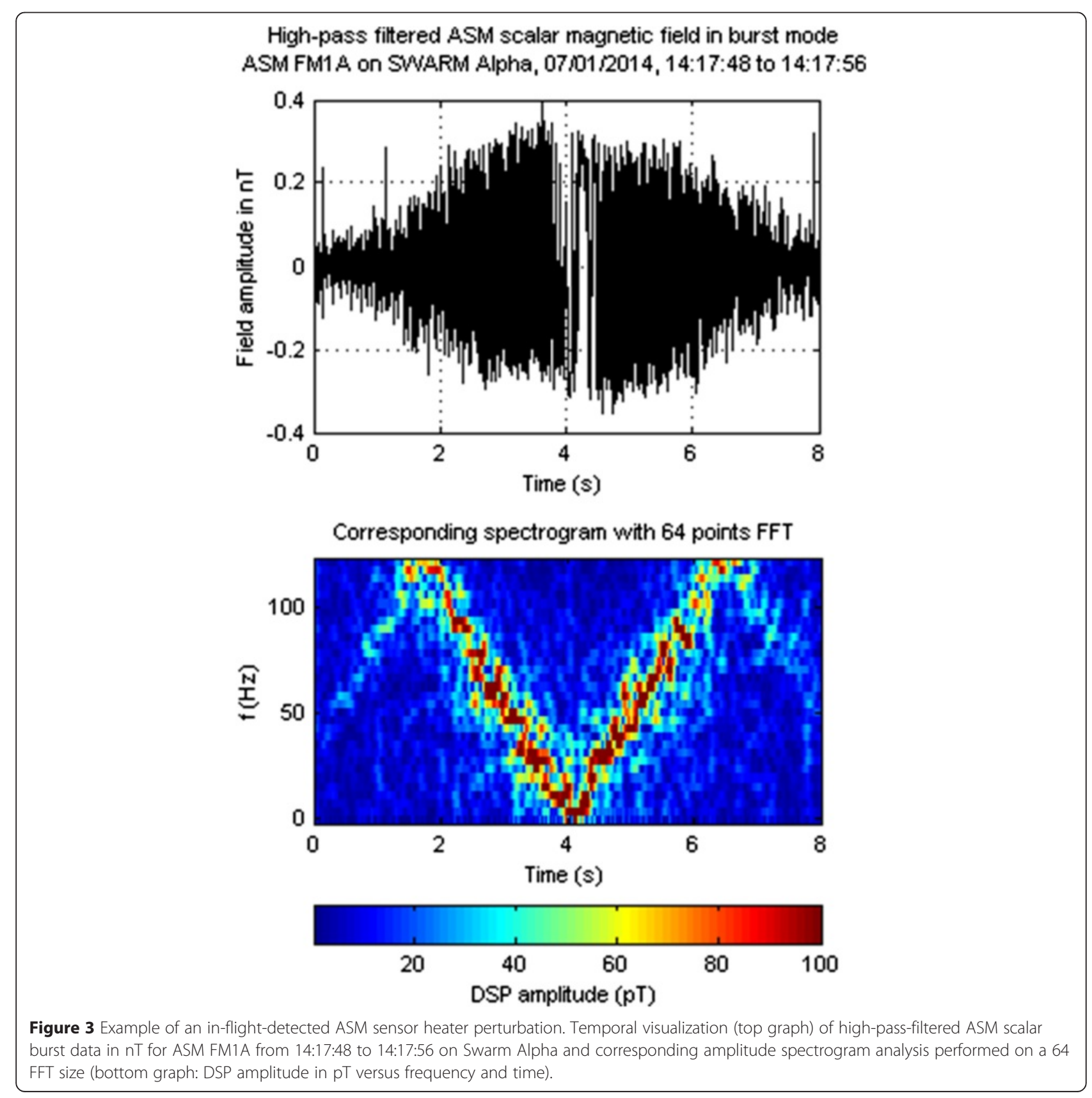




\section{Mean ASM scalar magnetic field measured on satellite Swarm Alpha and location of} the sensor heater perturbations from $01 / 12 / 2014$ to $01 / 01 / 2015$

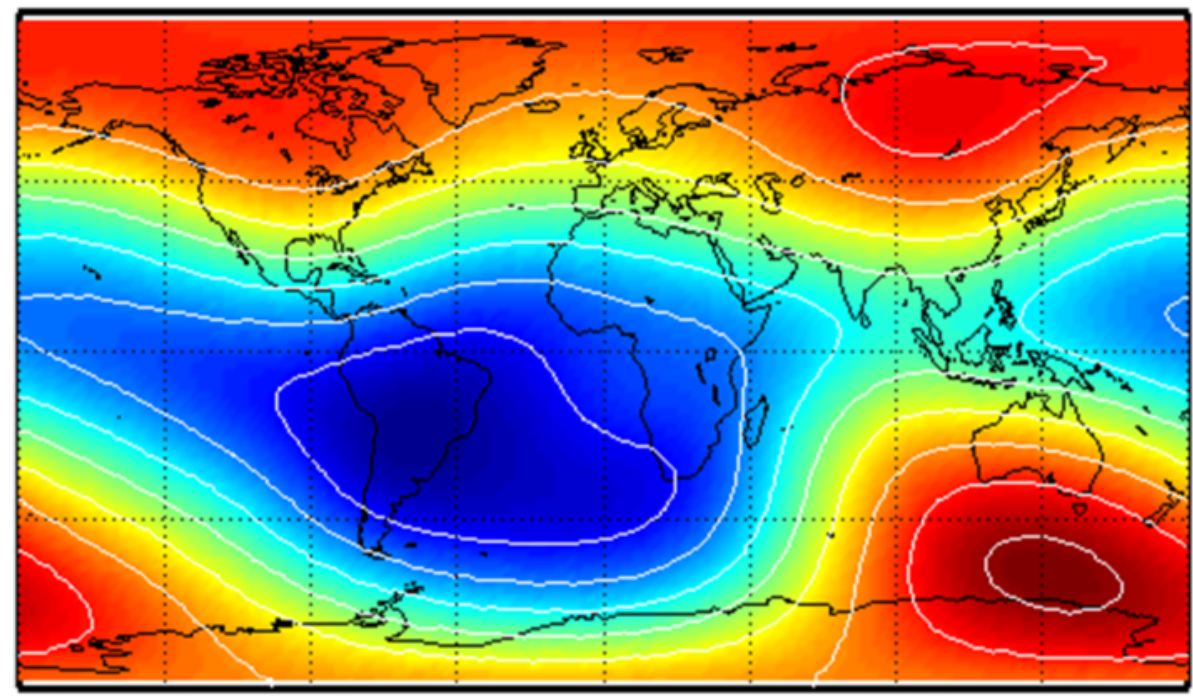

HF ASM sensor heater perturbations

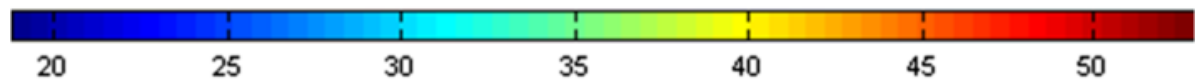

$(\mu \mathrm{T})$

Figure 4 Geographical distribution of locations where a heater's harmonics crosstalk perturbations may occur. This plot is for Swarm Alpha on December 2014. The ASM scalar field measurements are plotted in $\mu$ T (colorscale); possible perturbation locations are plotted as white lines.

interpolated data in the burst datasets. A noise spectrogram of such cleaned burst data is displayed in Figure 5 for a long acquisition period. As can be seen, only the previously detailed ASM RF modulation aliasing (around $30 \mathrm{~Hz}$ ) and ASM internal laser $D_{0}$ wavelength control loop (3.05 Hz and harmonics) signatures remain permanently, while noise bursts occur from time to time, with signatures on the spectrogram corresponding to vertical lines (mostly at high latitudes). A scalar noise analysis in the [35- to 40-Hz] frequency band performed on corrected burst mode noise spectrograms for all instruments during commissioning reveals scalar resolutions of respectively $1.1,1.0$, and $1.5 \mathrm{pT} / \mathrm{Hz}$ for the ASM nominal instruments on Swarm Alpha, Bravo, and Charlie, in full agreement with on-ground characterizations.

In addition to the flags previously defined for the detection of the ASM sensor head motor activations and the HF heater signal crosstalk perturbations, flags based on noise outliers have also been introduced. They point at field measurements outside the daily $\pm 3 \sigma$ range of high-pass-filtered scalar and vector measurements. These flags can advantageously be used as an additional criterion for ASM vector data selection.

\section{Preliminary vector calibration and vector mode performance assessment}

Taking advantage of the flags defined as explained above, the ASM vector mode calibration process and resulting performances could also be analyzed during the commissioning of the instruments. The three orthogonality angles characterizing the ASM's reference frame, defined by the orientation of the vector modulation coils and derived from the calibration algorithm, were found to be stable in the millidegree range around mean absolute values of a few tens of millidegrees respectively. The three derived vector modulation scale factors were checked to be close to $50 \mathrm{nT}$ for the $X$ - and $Z$-axis coils and close to $48 \mathrm{nT}$ for the $Y$-axis, in good agreement with the expected by-design values. A linear temperature coefficient of $47 \mathrm{ppm} / \mathrm{K}$ was estimated in flight, confirming on-ground measurements for each of the ASM axis transfer functions, again in agreement with the intrinsic properties of the amagnetic PEEK polymer used for the ASM sensor head. For corrections related to the rotor attitude, weighting correction factors in the 10-ppm range for the $\cos (\theta)$ and $\sin (\theta)$ corrections (sensor residual imperfections) and in the 70- to 80-ppm range for 


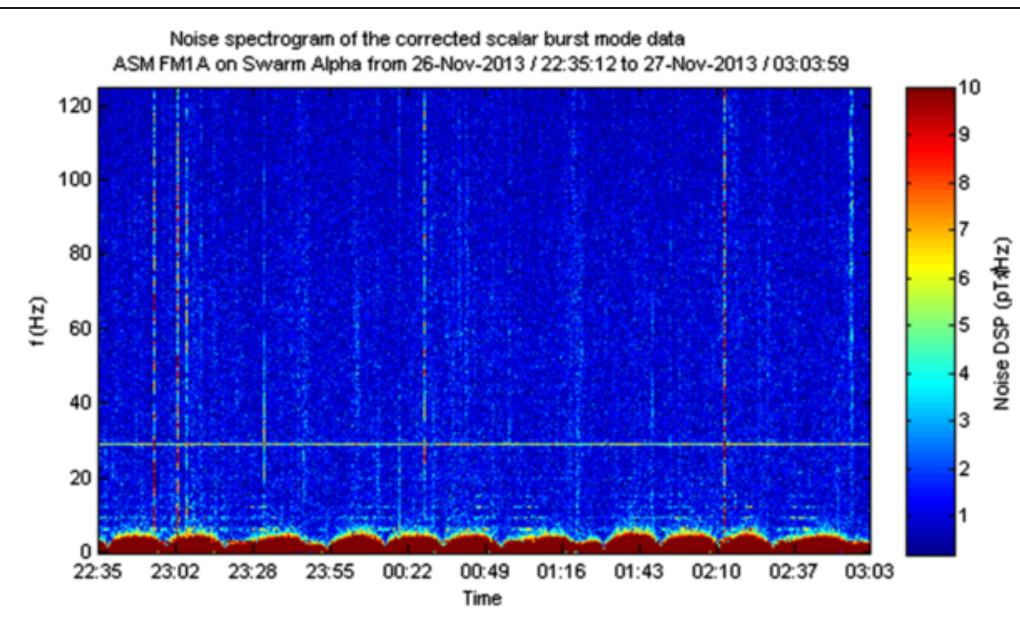

Figure 5 Noise spectrogram of corrected burst mode data. This spectrogram is for ASM FM1A on Swarm Alpha, corrected for motor activations and HF heater crosstalk perturbations. The spectrogram is computed with successive PSDs computed on a 2,048-point window (burst data sampling rate of $250 \mathrm{~Hz}$ ), with a $75 \%$ overlap between two consecutive windows. PSDs are plotted in $\mathrm{pT} / \sqrt{ } \mathrm{Hz}$ versus measurement time and frequency in $\mathrm{Hz}$.

$\cos (4 \theta)$ corrections (consequence of the coils geometry) have been determined. Since the beginning of the mission, the calibration process has been performed on a daily basis over a 3-day sliding window scheme. The corresponding daily vector calibration performance level is illustrated in Figures 6 and 7 for all ASM vector data on 3 June 2014, which is representative of the performance achieved.
The ASM scalar residual distribution characteristics of both raw and flag filtered vector measurements are shown in Figure 6. Without any specific data filtering, the scalar residuals of the ASM nominal instruments are characterized by a standard deviation $\sigma$ of respectively 2.3, 2.4, and $4.5 \mathrm{nT}$ on Swarm Alpha, Bravo, and Charlie. While nominal instruments on Swarm Alpha and Bravo
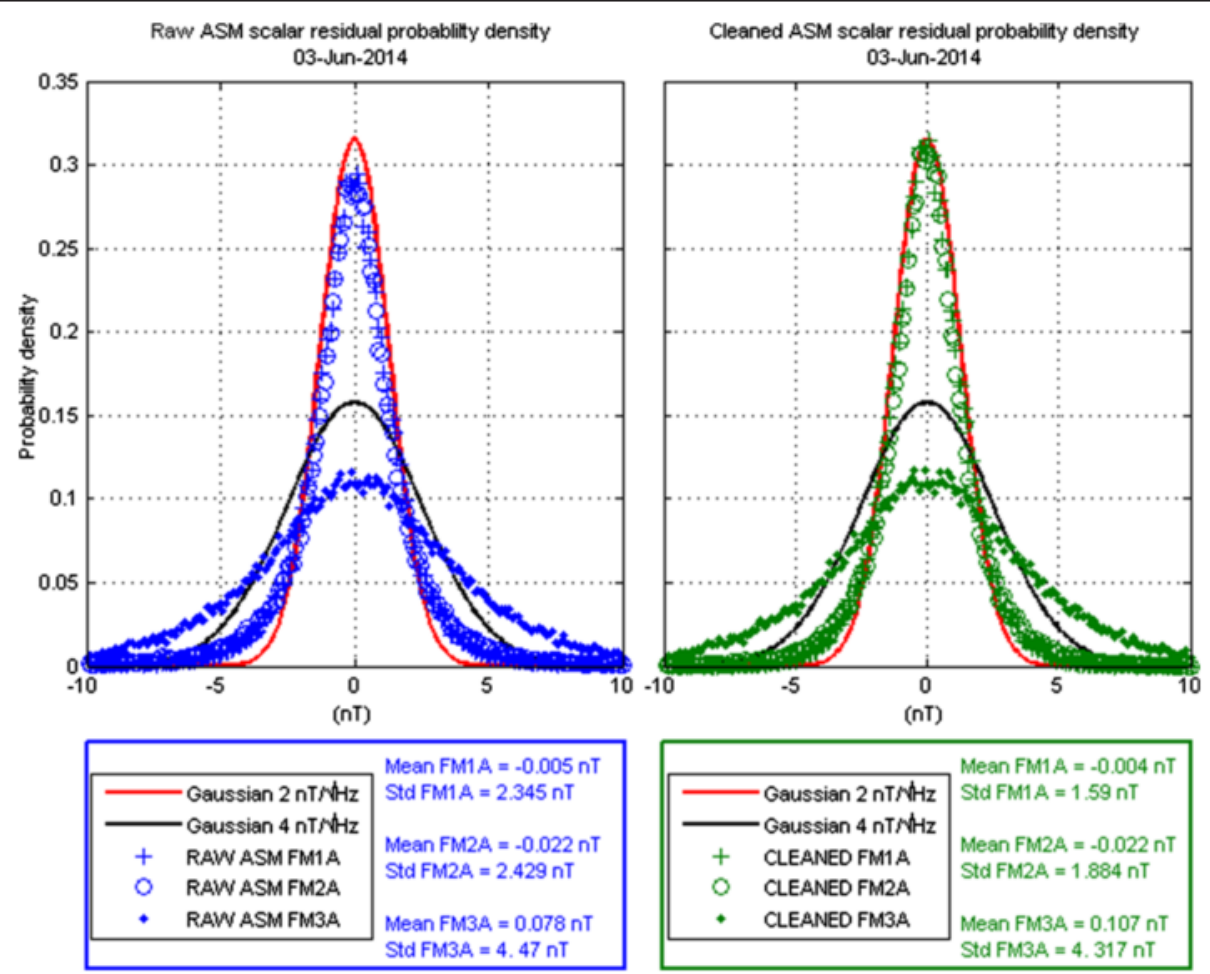

Figure 6 ASM scalar residual probability characteristics. Raw ASM data (left side) and flag-filtered data (right side) correspond to 3 June 2014 for all three satellites. The probability densities are plotted versus the scalar residuals (units in nT). 


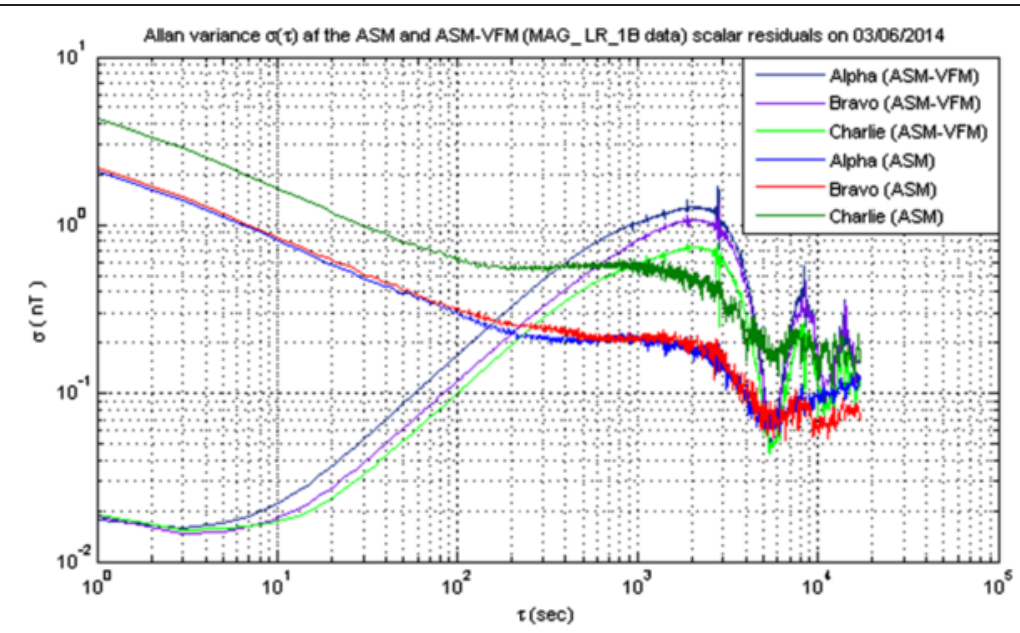

Figure 7 Allan variance analysis of daily scalar residuals. Comparison of the ASM and ASM-VFM scalar residuals by Allan variance analysis for Swarm Alpha, Bravo, and Charlie satellites. The Allan variance values are calculated from ASM and VFM 1-Hz data of 3 June 2014, given in nT for integration periods $\mathrm{t}$ between $1 \mathrm{~s}$ and $4.8 \mathrm{~h}$ (i.e., one fifth of a day).

exhibit similar vector noise characteristics, the one on Swarm Charlie shows almost twice as much noise, a difference that cannot just be attributed to the higher scalar noise level of this instrument compared to those of Alpha and Bravo. The flag-filtered data exhibit reduced $\sigma$ characteristics of respectively 1.6, 1.9, and $4.3 \mathrm{nT}$. For the ASM instruments on Swarm Alpha and Bravo, these characteristics are in close agreement with those expected, given the intrinsic ASM vector noise. For the instrument on Swarm Charlie, the flag-based data selection does not lead to much improvement, revealing a vector noise level significantly higher than expected, given the scalar noise level otherwise measured on this instrument. At this stage, no satisfactory explanation for this specific behavior has been found.

An interesting analysis of the scalar residual behavior can also be provided by the Allan variance (Allan 1966), which is a commonly used time domain analysis technique for assessing the stability of time references (clocks) and inertial sensors (e.g., accelerometers and gyroscopes). It consists in representing the root mean square random drift errors of a measurement as a function of averaging times. It thus provides information on the nature and magnitude of various sensor noise and drift terms. Figure 7 displays the Allan variance analysis of the ASM scalar residuals, which we also compare to the ASM-VFM scalar residuals, defined as the difference between the ASM scalar measurements and the modulus of the calibrated VFM vector data extracted from the official Swarm Mag_LR_1B product (dataset '03'). First considering ASM scalar residuals, we can see that for low time average periods, between 1 and $100 \mathrm{~s}$, the Allan variance exhibits a $\tau^{-1 / 2}$ drift typical of a white noise-driven behavior, with values $\sigma$ for $\tau=1 \mathrm{~s}$ corresponding to the vector resolutions in $\mathrm{nT} / \sqrt{ } \mathrm{Hz}$. For $\mathrm{\tau}$ higher than $200 \mathrm{~s}$, the ASM instruments show mid-term (successively suborbital and then orbital) vector bias stability in the 0.2-nT range on Swarm Alpha and Bravo and in the 0.5- to 0.6-nT range on Swarm Charlie. No specific remaining periodic signature is otherwise detected in any of the three ASM scalar residuals, and daily orbital stability figures are well within the expected ASM vector performance, with error characteristics significantly lower than $1 \mathrm{nT}\left(\begin{array}{ll}1 & \sigma\end{array}\right)$. Turning to ASM-VFM scalar residuals reveals much smaller variance values for short periods of time. This was expected since the VFM instruments exhibit a much lower vector noise level $\quad(11 \mathrm{pT} / \sqrt{ } \mathrm{Hz}$ at $1 \mathrm{~Hz})$ than the ASM vector mode. But it also reveals a much degraded stability, compared to the ASM residuals, for $\tau$ higher than $200 \mathrm{~s}$. This unexpected disturbance on the VFM measurements is being investigated by an ESA-lead Swarm-wide team. The investigations currently favor Sun-driven perturbation effects in the vicinity of the VFM sensor.

Encouraged by these first promising ASM vector mode performances, data have since been systematically acquired and a self-consistent and autonomous ASM $1-\mathrm{Hz}$ vector product, referred to as ASM_V, has been produced. This product is elaborated on the basis of the Mag_LR_1B product, where VFM data are replaced by ASM vector data. The product is computed and generated on a daily basis with a 3-day sliding window scheme for the ASM vector calibration parameters. The corresponding daily-calibrated ASM vector measurements are then provided in both the ASM and VFM reference frames, thanks to an ASM to VFM rotation matrix also computed periodically.

\section{ASM long-term vector performance analysis}

To further improve our knowledge of the ASM in-flight behavior, the vector measurements have also been 
analyzed over long time periods to check whether observed variations could be correlated with some evolution in the instrument's parameters.

\section{Performance of the daily-calibrated and computed ASM_V product}

The long-term performance of the daily-calibrated ASM vector data has first been evaluated through the analysis of the ASM_V scalar residual depicted in Figure 8. This figure shows the daily mean and standard deviation values of the raw 1-Hz ASM_V data between the beginning of the mission and the end of 2014, amounting to slightly more than 1 year of data ${ }^{\mathrm{a}}$. The overall vector performances are consistent with all raw ASM vector data characteristics previously discussed. Similar performances are demonstrated in the nominal ASM instruments on Swarm Alpha and Bravo, with daily mean values well within the \pm 20 -pT range and daily standard deviations in the [2- to $2.5-\mathrm{nT}]$ range. It is worth noting, however, that these results are degraded during satellite maneuvers, when the ASM instrument thermal equilibrium is temporarily modified with characteristic times of at least several hours, affecting the vector calibration process. To avoid such issues, the use of data subsets shorter than 1 day to perform several consecutive vector calibrations instead of a single one and compensate for maneuver effects would be a possibility. But this turns out to not be very robust, as the data used in the calibration process need to cover a wide enough range of field orientations and field amplitudes. Finally, we note that on Swarm Charlie, the means of the raw scalar residuals lie in the \pm 40 -pT range while their standard deviations are in the [4- to 6-nT] range, in agreement with the high ASM vector noise behavior previously highlighted on Charlie.

It also is worth pointing out that these scalar residual characteristics do not exhibit any unexpected geographical signature. This is illustrated in Figure 9, in which geographical means and standard deviations of the ASM_V scalar residuals for all three satellites in June 2014 are displayed. The geographical means show uniform behavior while, as expected, the geographical standard deviations are proportional to the ambient scalar magnetic field modulus. The daily-calibrated ASM-V products thus provide long-term ASM vector measurements in line with ASM vector performance demonstrated on-ground. These data have already been successfully used for geomagnetic field modeling studies (Hulot et al. 2015, Vigneron et al. 2015).

\section{Towards a single and unique vector calibration}

Finally shifting to the engineer's point of view, one can also look into the long-term evolution of the instrument
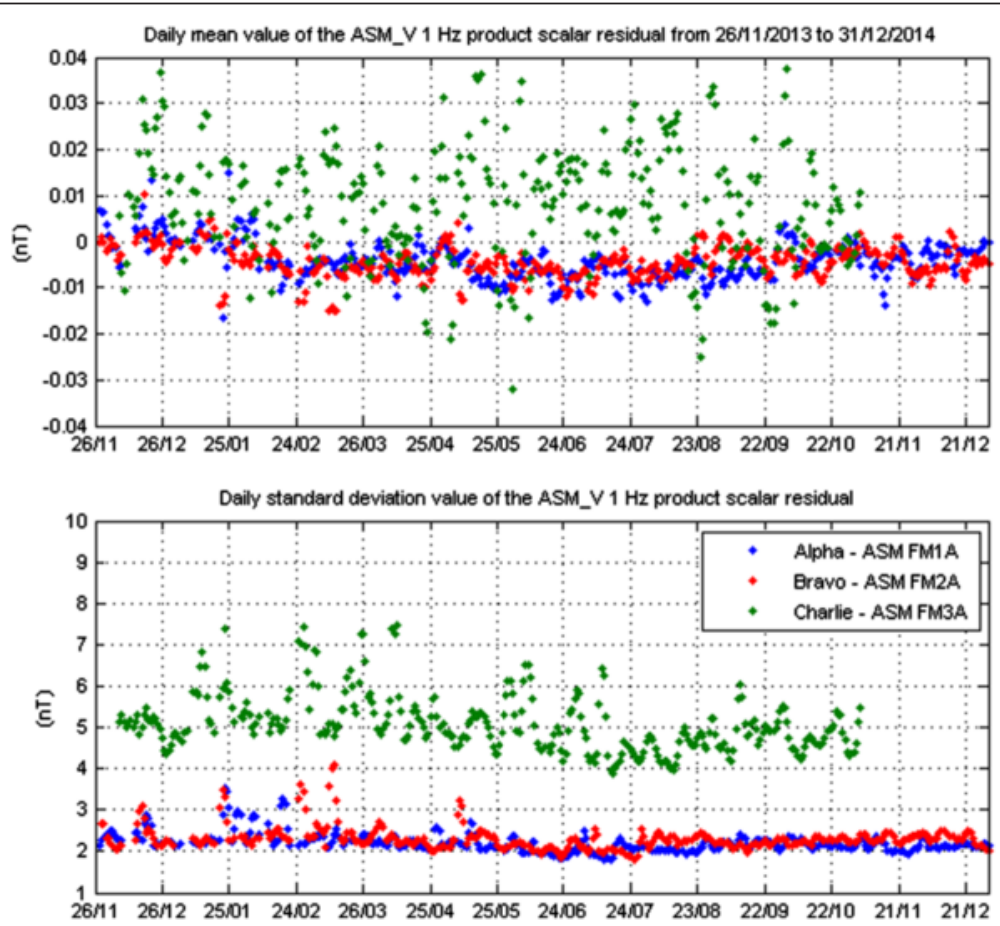

Figure 8 Daily mean and standard deviation values of ASM_V scalar residuals. Values are given in nT from 1 January 2014 to 31 December 2014 No flag filtering is applied on the ASM_V data. The specific/noisier behavior of the ASM FM3A instrument on Swarm Charlie is very clear (until the anomaly of 5 November 2014 and the subsequent loss of ASM vector data). Note also the peaks in the standard deviations of Alpha and Bravo, corresponding to times of satellite maneuvers during commissioning. 


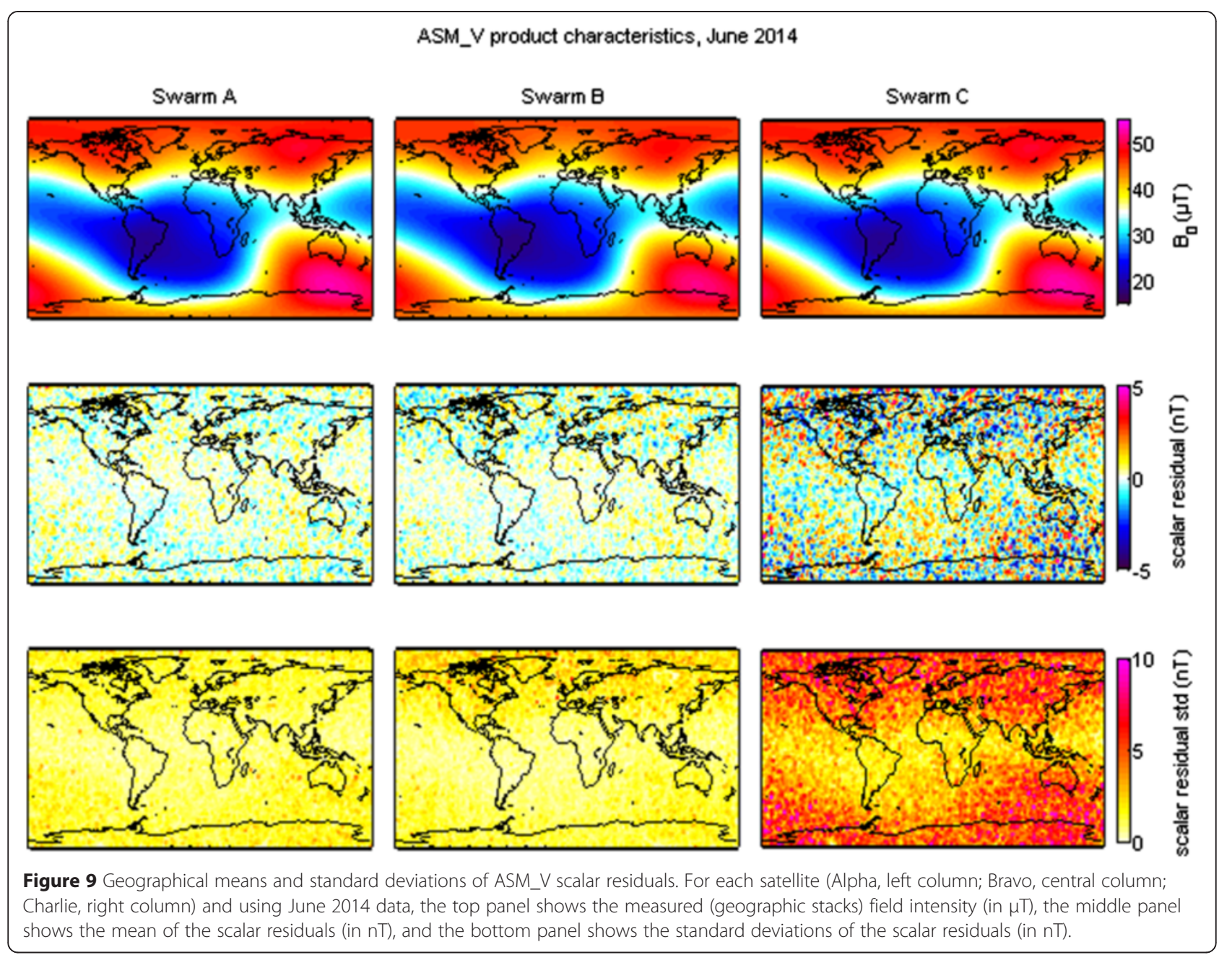

parameters to gain a better understanding of the ASM's behavior. This can first be performed by analyzing the daily evolution of the ASM vector calibration parameters. After more than 1 year of data, most of the vector calibration parameters, such as vector coil orthogonality angles and linear temperature expansion correction coefficients, have been found to be very stable. These could easily be set to constant values in the daily vector calibration process. The daily-computed orthogonality angles, for instance, have been found to display a standard deviation of less than 1 millidegree over more than 1 year of data. In contrast, a long-term seasonal modulation is clearly detected when looking at the vector modulation scale factors, as depicted in Figure 10. This behavior is seen in similar ways in all ASM instruments, though with less accuracy on the $Y$-axis, since this axis is most of the time almost perpendicular to the ambient magnetic field direction. As a consequence, vector field reconstructions performed with a single set of calibration parameters (ignoring seasonal evolutions) would exhibit long-term drifts. This, in fact, is exactly the reason why it was decided to proceed with daily calibrations up to now. Unfortunately, this seasonal behavior is not directly correlated to any of the ASM internal housekeeping observables. Correcting for it thus requires that we look for additional inputs, not directly available at the ASM level. A closer look at the long-term evolution of the satellites' orbital characteristics (e.g., satellite and ASM reference frame attitude w.r.t Sun illumination, which are suspected to be at the origin of these evolutions) as well as at closely correlated platform housekeeping parameters (e.g., external temperature measurements and daily duty cycle of the ASM heaters) reveals some correlations with the evolutions depicted in Figure 10, as illustrated in Figure 11. The benefit of taking into account these additional parameters as extra first-order corrections (implemented in the same way as the corrections previously detailed) on the ASM vector scale factors has been considered and is illustrated in Figure 12. This figure compares ASM scalar residuals obtained with daily calibration (ASM_V data product) versus single calibrations performed with or without external inputs to compensate 

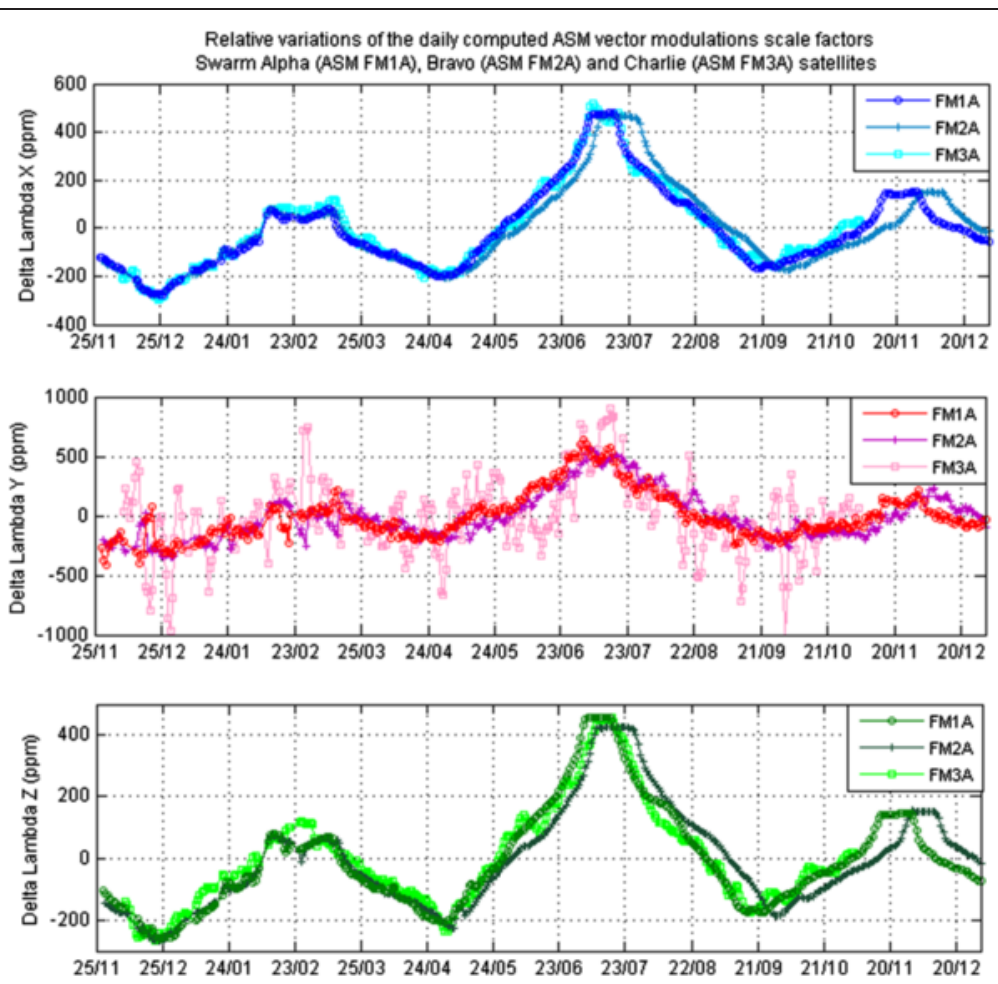

Figure 10 Long-term relative variations of ASM vector modulation scale factors. Relative evolutions in ppm with respect to the mean global values of the daily computed vector modulation scale factors for all ASM nominal instruments on Swarm Alpha, Bravo, and Charlie satellites from 1 January 2014 to 31 December 2014.
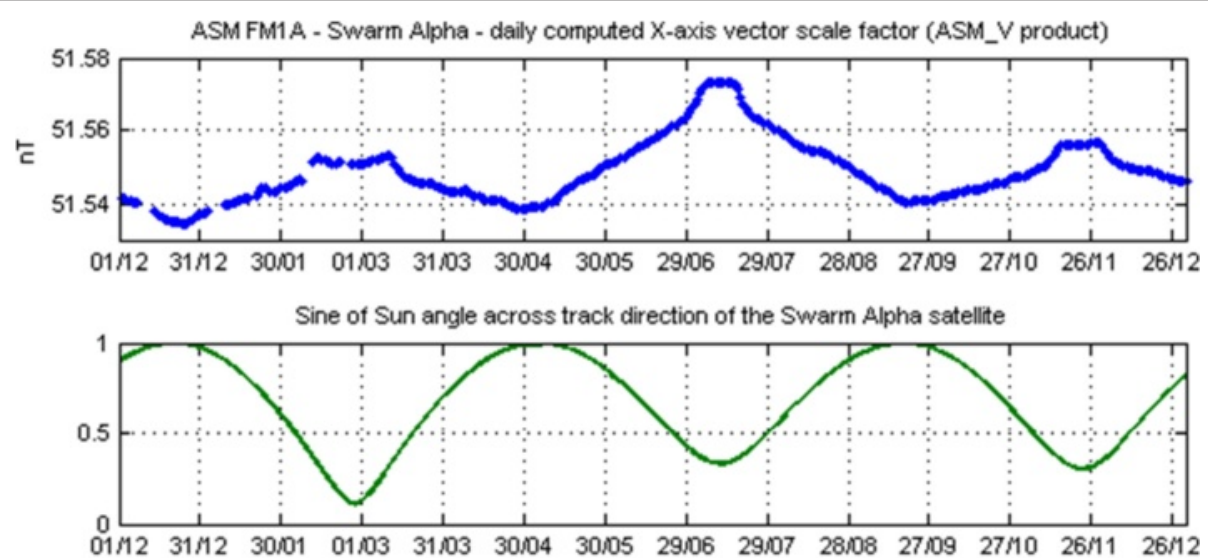

Daily computed ASM sensor heater duty-cylcle (from Swarm Alpha satellite plateform housekeeping)

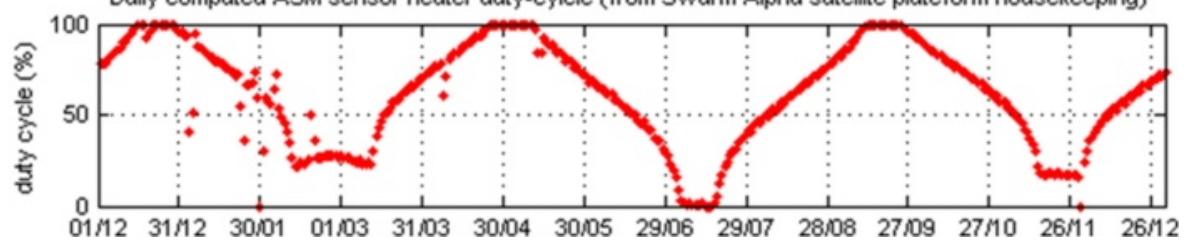

Figure 11 Correlation of external parameters with ASM vector scale factors. To illustrate the possible influence of external factors on the ASM vector parameters of Swarm Alpha (here ASM FM1A X-axis vector scale factor in $\mathrm{nT}$, reproduced in the upper graph), the second and third graphs respectively display the evolution of the sine of the Sun illumination angle along orbit track in the ASM reference frame (potential origin of the seasonal effect) and of the in-flight ASM heater duty cycle in percent (testifying for the evolution in the thermal conditions witnessed by the ASM sensor) from 1 January 2014 to 31 December 2014. 

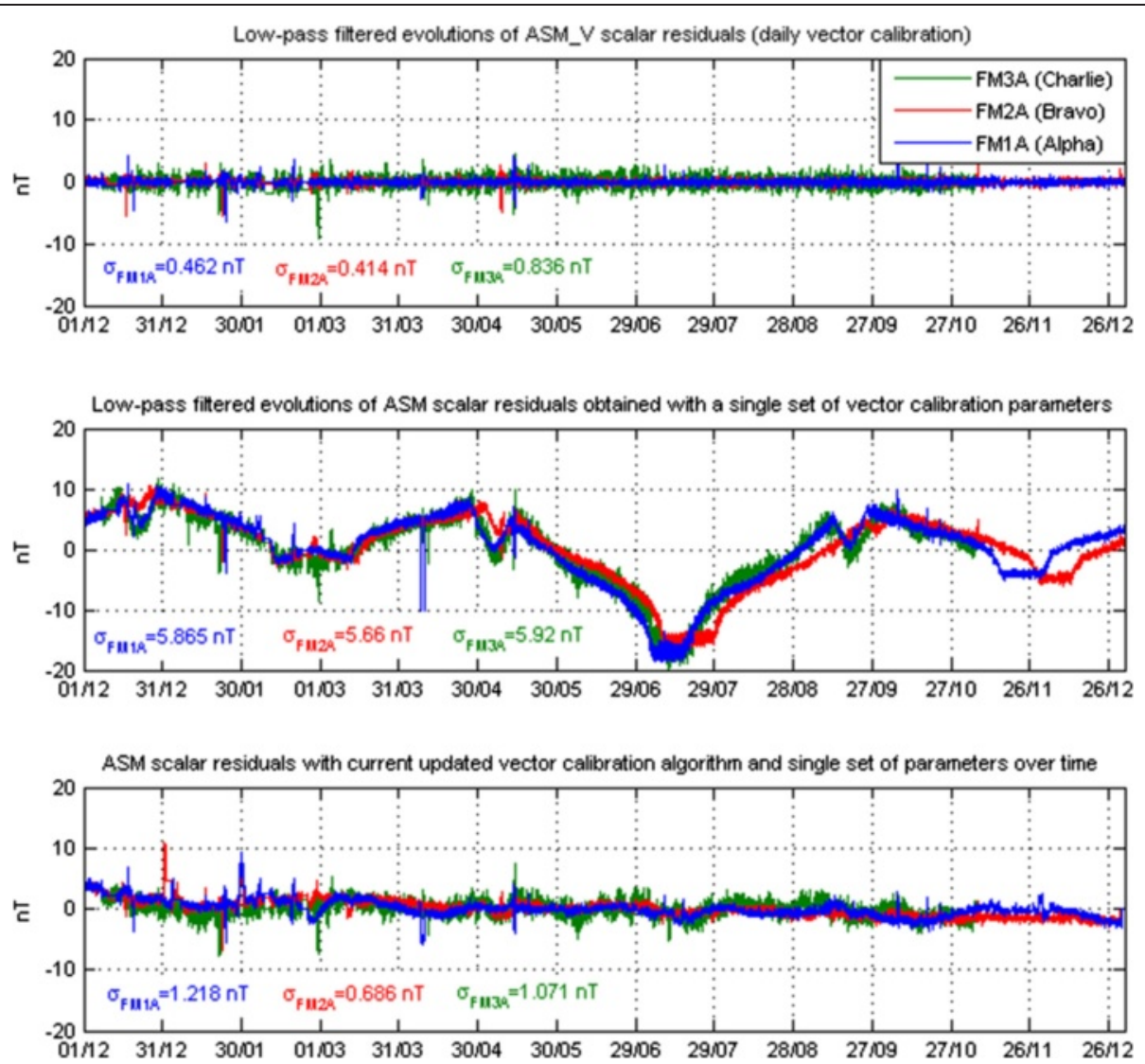

Figure 12 Single versus daily vector calibration process. The upper graph displays the low-pass-filtered ASM scalar residuals obtained with the ASM-V product (daily vector calibration), while the lower ones display the ASM vector data using the same vector algorithm with a single and constant set of vector calibration parameters without (middle graph) and with (bottom graph) external inputs for the correction of seasonal effects. All residuals are plotted in nT from 1 January 2014 to 31 December 2014.

for the seasonal effects. A major improvement is demonstrated in the evolution of the data taking into account external inputs: a reduction by a factor of 5 in amplitude is found compared to the long-term stability characteristics of the single calibrated data without external inputs. The linear corrections with respect to these external parameters, to be applied to the vector scale factors and that are currently being investigated and implemented, clearly point at thermal gradient-related effects subject to both spatial and temporal variations. These effects would affect the mean shape of the ASM vector coils and, as a consequence, their transfer functions. While these first results look promising, there clearly is still room for additional improvements to achieve long-term performance equivalent to that provided by the daily calibration of the ASM-V product (a remaining factor 2 improvement on the scalar residual characteristics of the single calibrated data with external inputs is still to be gained). Studies are ongoing to refine the present model. The progressive drifts of the satellites' local times, combined with the extended time coverage, will hopefully provide further valuable insight into these effects and allow for their prediction (in particular, semi-annual and annual effects should progressively be highlighted after a few years of mission).

\section{Conclusion}

In-flight ASM vector mode performance could be assessed during both commissioning and operational phases. This mode, initially developed on an optional and experimental basis, has now become the nominal operation mode of all ASM instruments on board the Swarm satellites. In addition to the high accuracy and high-resolution scalar measurements nominally delivered for in-flight calibration of the VFM instrument, this vector mode can also deliver fully consistent vector measurements by taking advantage of both the internal high scalar bandwidth and the high resolution of the ASM sensor. It displays characteristics in excellent agreement with on-ground characterizations and ASM specifications for the mission. This is very encouraging, given that simultaneous availability of scalar and vector measurements using a single instrument is a world first for space magnetometers. It should open interesting possibilities for future missions based on space magnetometry. 
Encouraged by these performances, a daily vector calibrated ASM_V product has been generated for scientific exploitation. Its long-term behavior has been investigated in connection with both instrumental and satellite platform environment parameter evolutions. This ASM_V product also made it possible to evaluate the in-flight mechanical boom stability between the ASM and VFM instruments (Fratter et al. 2015). Scientific exploitation of the data has also started. In particular, a geomagnetic field model only relying on ASM data could be produced (Hulot et al. 2015), as well as an IGRF candidate model (Vigneron et al. 2015), which contributed to the final IGRF-12 model for 2015 to 2020 (Thébault et al. 2015), demonstrating the scientific interest generated by the vector measurements provided by the ASM instruments.

Last but not least, it should be noted that the three satellites of the Swarm mission are the first satellites to offer the possibility of comparing vector measurements delivered by two different types of instruments in space. This specific and unique capability can be used to directly crosscheck, compare, and assess the relative sensors' vector performance and to build and validate geophysical models with different input datasets.

\section{Endnotes}

${ }^{\text {a }}$ Please note that for all long-term ASM data evaluations, no ASM data is available on the Swarm Charlie satellite since 5 November 2014 when an ASM instrument anomaly occurred. This event is currently under investigation and prevents any new ASM data delivery on Swarm Charlie.

\section{Competing interests}

The authors declare that they have no competing interests.

\section{Authors' contributions}

J-ML and TJ drafted the manuscript and subsequent revisions. TJ has developed and upgraded the ASM vector calibration algorithm for both ASM_V product and long-term analysis with a single calibration objective. All authors have contributed to the analysis of the ASM data, from the commissioning of the instruments up to the vector mode data analysis during exploitation. All authors read and approved the final manuscript.

\section{Acknowledgements \\ The authors acknowledge the ESA Swarm team for computing and providing additional data access on Swarm satellites orbital characteristics for the long-term analysis of the ASM instrument behavior. They also gratefully acknowledge support from the Centre National d'Études Spatiales (CNES) within the context of the Travaux préparatoires et exploitation de la mission SWARM' project. This is IPGP contribution no. 3628.}

\section{Author details}

'Université Grenoble Alpes, F-38000 Grenoble, France. ${ }^{2}$ CEA, LETI, MINATEC Campus, F-38054 Grenoble, France. ${ }^{3}$ Institut de Physique du Globe de Paris, Sorbonne Paris Cité, Université Paris Diderot, CNRS, F-75005 Paris, France. ${ }^{4}$ Cooperative Institute for Research in Environmental Sciences, University of Colorado, Boulder, USA. ${ }^{5}$ NOAA National Centers for Environmental Information (NCEI), Boulder, Colorado, USA. ${ }^{6}$ Centre National d'Etudes Spatiales (CNES), Toulouse, France.
Received: 6 February 2015 Accepted: 15 April 2015

Published online: 25 April 2015

\section{References}

Allan DW (1966) Statistics of atomic frequency standards. Proc IEEE 54-2:221-230 Fratter I, Léger JM, Bertrand F, Jager T, Hulot G, Brocco L, Vigneron P (2015)

Swarm absolute scalar magnetometers first in-orbit results. Astronautica, Acta, in review

Friis-Christensen E, Lühr H, Hulot G (2006) Swarm: a constellation to study the Earth's magnetic field. Earth Planets Space 58:351-358

Gravrand O, Khokhlov A, Le Mouël JL, Léger JM (2001) On the calibration of a vectorial ${ }^{4} \mathrm{He}$ pumped magnetometer. Earth Planets Space 53:949-958

Guttin C, Léger JM, Stoeckel F (1994) An isotropic earth field scalar magnetometer using optically pumped helium 4. J de Physique IV 4:655-659

Hulot G, Vigneron P, Léger JM, Fratter I, Olsen N, Jager T, Bertrand F, Brocco L, Sirol O, Lalanne X, Boness A, Cattin V (2015) Swarm's absolute magnetometer experimental vector mode, an innovative capability for space magnetometry. Geophys. Res. Lett., 42, doi:10.1002/2014GL062700

Jager T, Léger JM, Bertrand F, Fratter I, Lalaurie JC (2010) SWARM Absolute Scalar Magnetometer accuracy: analyses and measurement results. Proceedings of the IEEE Sensors 2010 conference 2392-2395

Kernevez N, Duret D, Moussavi M, Léger JM (1992) Weak field NMR and ESR spectrometers and magnetometers. IEEE Transactions on Magnetics 28-5:3054-3059

Léger JM, Bertrand F, Jager T, Prado ML, Fratter I, Lalaurie JC (2009) Swarm absolute scalar and vector magnetometer based on helium 4 optical pumping. Procedia Chemistry 1:634-637

Léger JM, Bertrand F, Jager T, Fratter I (2011) Spaceborn scalar magnetometers for Earth's field studies. Proceedings of the 62nd International Astronautical Congress, Cape Town, South Africa, Oct. 3-7, 2011, paper IAC-11-B1.3.9.

Thébault E, Finlay CC, Beggan C, Alken P, Aubert J, Barrois O, Bertrand F, Bondar T, Boness A, Brocco L, Canet E, Chambodut A, Chulliat A, Coïsson P, Civet F, Du A, Fournier A, Fratter I, Gillet N, Hamilton B, Hamoudi M, Hulot G, Jager T, Korte M, Kuang W, Lalanne X, Langlais B, Léger JM, Lesur V, Lowes FJ et al (2015) International Geomagnetic Reference Field: the twelfth generation. Space, Earth Planet, in press

Vigneron P, Hulot G, Olsen N, Léger JM, Jager T, Brocco L, Sirol O, Coïsson P, Lalanne X, Chulliat A, Bertrand F, Boness A, Fratter I (2015) A 2015 IGRF candidate model based on Swarm's experimental ASM vector mode data. Space, Earth Planet, in review

\section{Submit your manuscript to a SpringerOpen ${ }^{\odot}$ journal and benefit from:}

- Convenient online submission

- Rigorous peer review

- Immediate publication on acceptance

- Open access: articles freely available online

- High visibility within the field

- Retaining the copyright to your article

Submit your next manuscript at $\boldsymbol{~ s p r i n g e r o p e n . c o m ~}$ 